\title{
Inner surface enhanced femtosecond second harmonic generation in thin $\mathrm{ZnO}$ crystal tubes
}

\author{
C. C. Zheng, ${ }^{1}$ S. J. Xu, ${ }^{1, a)}$ J. Q. Ning, ${ }^{1}$ S. F. Zhang, ${ }^{2}$ J. Y. Wang ${ }^{2}$ C. M. Che ${ }^{3}$ and \\ J. H. $\mathrm{HaO}^{4}$ \\ ${ }_{1}^{1}$ Department of Physics and HKU-CAS Joint Laboratory on New Materials, The University of Hong Kong, \\ Pokfulam Road, Hong Kong, China \\ ${ }^{2}$ State Key Laboratory of Crystal Materials, Shandong University, Jinan 250100, China \\ ${ }^{3}$ Department of Chemistry, Institute of Molecular Functional Materials, HKU-CAS Joint Laboratory \\ on New Materials, The University of Hong Kong, Pokfulam Road, Hong Kong, China \\ ${ }^{4}$ Department of Applied Physics, The Hong Kong Polytechnic University, Hung Hom, Hong Kong, China
}

(Received 12 June 2010; accepted 29 November 2010; published online 14 January 2011)

\begin{abstract}
At room temperature, efficient second harmonic generation (SHG) emission is observed in thin $\mathrm{ZnO}$ crystal hollow rods (tubes) with diameter $\sim 0.2 \mathrm{~mm}$ under the excitation of femtosecond laser from 700 to $860 \mathrm{~nm}$. Power and polarization dependence of the SHG signal on the primary excitation beam is also measured. The multiple total reflections between the outer and inner surfaces of the sample are analyzed to be responsible for the efficient SHG. Ninefold amplification of SHG signal in the tube structure is estimated. (C) 2011 American Institute of Physics. [doi:10.1063/1.3531566]
\end{abstract}

\section{INTRODUCTION}

Recently, $\mathrm{ZnO}$ has attracted an intensive interest as a promising material for fabricating efficient ultraviolet lightemitting devices operating at room temperature and above, as well as for potential applications in nonlinear optics, spintronics, and green energy, due to its large direct band gap, big exciton binding energy, and other outstanding properties. ${ }^{1}$ Most of the previous works about the optical properties of $\mathrm{ZnO}$ with various morphologies were conducted in the linear area. ${ }^{2-5}$ More recently, the nonlinear optical properties of $\mathrm{ZnO}$, for instance, second harmonic generation (SHG), ${ }^{6-10}$ third harmonic generation, ${ }^{11,12}$ and multiphoton absorption (MPA) induced luminescence, ${ }^{13-15}$ have been investigated. However, relatively weak SHG emission to the band edge emission was observed in singlecrystal $\mathrm{ZnO}$ bulk. ${ }^{14}$ In this paper, an extraordinary strong SHG emission compared to the band edge emission was observed in a thin $\mathrm{ZnO}$ hollow crystal rod under the excitation of femtosecond laser from 700 to $860 \mathrm{~nm}$ at room temperature. The distinct hollow geometric structure of the sample is believed to be responsible for this enhanced SHG emission.

\section{SAMPLE AND EXPERIMENTAL DETAILS}

The samples used in this study are the wurtzite $\mathrm{ZnO}$ single-crystal rods with diameters of $\sim 0.2 \mathrm{~mm}$ and lengths of $\sim 2-5 \mathrm{~mm}$. They were grown in a silver crucible by spontaneous nucleation method using hydrous $\mathrm{KOH}$ melt as flux. ${ }^{16}$ Interestingly, central hollow structures form inside these thin rods. X-ray diffraction, confocal micro-Raman and low-temperature photoluminescence measurements consistently indicate high quality of the samples. ${ }^{17,18}$ For the SHG measurements, wavelength tunable femtosecond laser beam with a $\sim 0.1 \mathrm{~mm}$ focus waist was focused onto the surface of the rods. The femtosecond pulse was generated from a self-

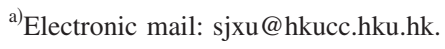

mode-locked Ti:sapphire oscillator (Tsunami) pumped by a $10 \mathrm{~W}$ solid-state laser (Millennia). It has a $\sim 80 \mathrm{fs}$ pulse width and a repetition rate of $82 \mathrm{MHz}$. Emission signals from the samples were collected by a pair of lenses, analyzed by Acton SP305 monochromator, and detected with a Hamamatsu photomultiplier tube (R928). Both the excitation light beam and collection direction of emission signal were perpendicular to the c-axis of hollow rods. The angle between the excitation light beam and emission collection direction is $\sim 26^{\circ}$. A standard lock-in amplification technique was used to improve the signal-to-noise ratio. A half-wave plate was employed to rotate the polarization direction of the excitation wave. X-ray diffraction experiments were performed on an x-ray diffractometer (XRD) (Model D8 Advance, Bruker), with $2 \theta$ ranging from $30^{\circ}$ to $80^{\circ}$. The Raman spectra and images were measured under a backscattering geometric configuration using WITec-Alpha confocal microRaman system. The scanning electron microscopic (SEM) image was acquired by using JEOL (JSM-7001F) SEM. All measurements were carried out at room temperature.

\section{RESULTS AND DISCUSSION}

Figure 1(a) depicts the x-ray diffraction patterns (XRD) of one sample, whereas its Raman scattering spectrum is shown in Fig. 1(b). Both the XRD and Raman data show that the sample has an excellent wurtzite crystalline structure. ${ }^{17}$ Figure 2 shows representative SHG emission (solid line) of the thin $\mathrm{ZnO}$ crystal rod under excitation by femtosecond laser with wavelength of $774 \mathrm{~nm}$ and power of $700 \mathrm{~mW}$ at room temperature. For comparison, a thick $\mathrm{ZnO}$ single crystal solid rod (diameter $\sim 3 \mathrm{~mm}$ ) was measured under the same excitation conditions and its emission spectrum (dashed line) was also shown. From the spectra in Fig. 2, a strong SHG emission at $387 \mathrm{~nm}$ was observed from the thin hollow rod, while a strong band-edge emission at $\sim 397 \mathrm{~nm}$ and a broad visible emission band were observed from the thick solid rod. It is clear that the SHG emission from the 

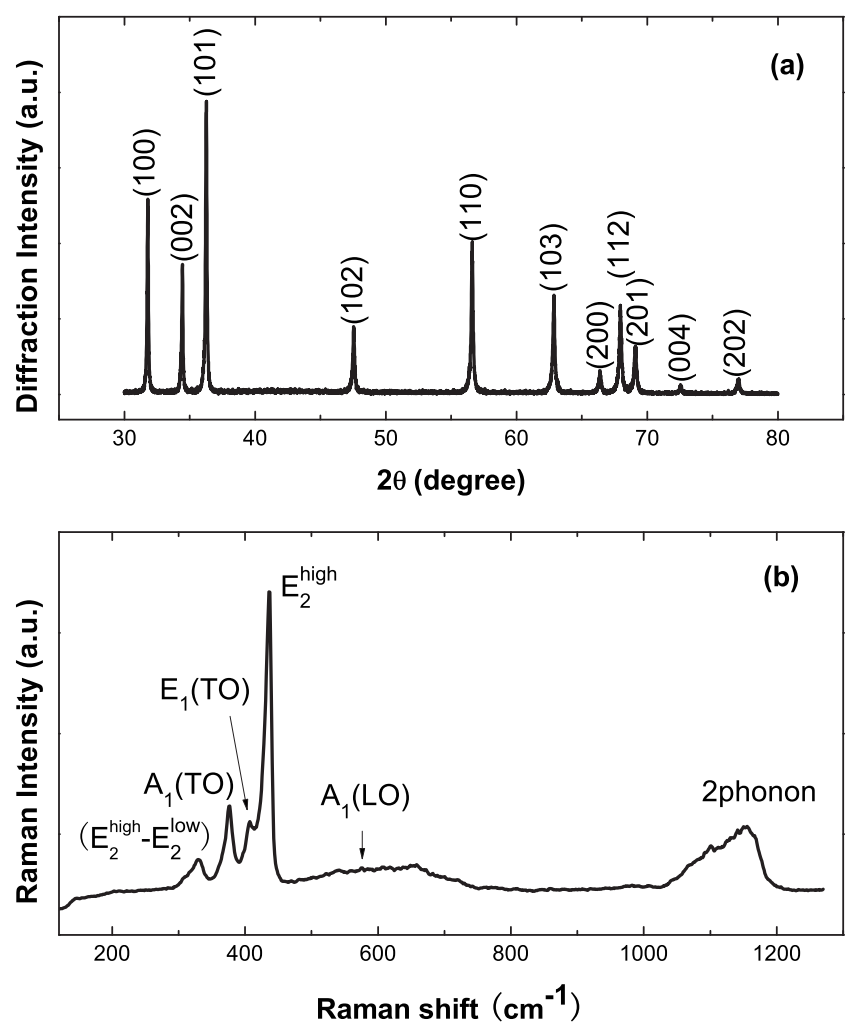

FIG. 1. (a) X-ray diffraction pattern of the thin $\mathrm{ZnO}$ crystal rod, indicating a well wurtzite crystallized structure. (b) Raman scattering spectrum of the thin $\mathrm{ZnO}$ crystal rod, which coincides with pervious work about $\mathrm{ZnO}$ bulk material.

thin $\mathrm{ZnO}$ hollow rod is very efficient. A schematic diagram of the illumination-collection geometric configuration undertaken in this study is shown in the inset of Fig. 2, in which $\mathrm{S}$ represents the sample holder surface, $\mathrm{z}$ : the c-axis of the sample, $\mathrm{x}$ : the emission collection direction, $\omega_{0}$ : the incident excitation light beam, $\mathrm{p}$ : the polarization direction of excita-

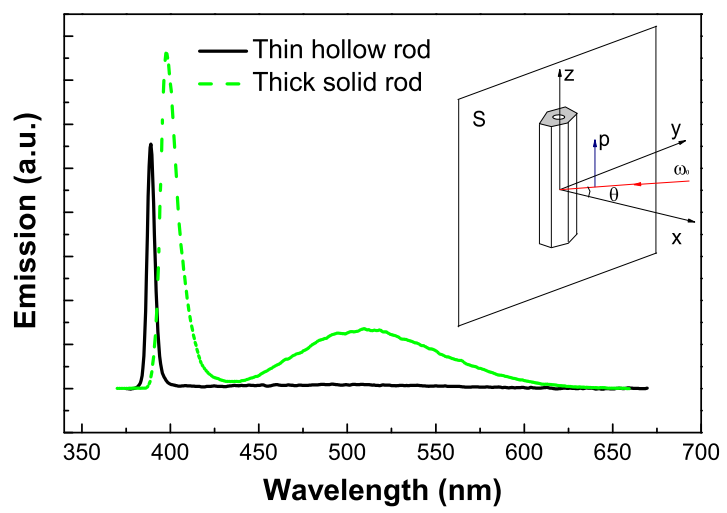

FIG. 2. (Color online) Emission spectra of a thin $\mathrm{ZnO}$ hollow rod (solid curve) and a thick $\mathrm{ZnO}$ solid rod (dashed curve) under the excitation of 774 $\mathrm{nm}$ femtosecond laser with power of $700 \mathrm{~mW}$ at room temperature. An enhanced SHG signal at $387 \mathrm{~nm}$ and MPA induced band-edge luminescence centered at $\sim 397 \mathrm{~nm}$ are observed from the thin hollow rod and thick solid rod, respectively. A broad visible band is also observed in the thick $\mathrm{ZnO}$ rod while it was much weaker in the thin hollow sample. The inset shows a schematic diagram of the illumination-collection geometric configuration undertaken in this study. S: the sample holder surface, $\mathrm{z}$ : the c-axis of the sample, $\mathrm{x}$ : the emission collection direction, $\omega_{0}$ : the incident excitation light beam, $\mathrm{p}$ : the polarization direction of excitation light, and $\theta$ : the angle between the excitation light beam and emission collection direction.

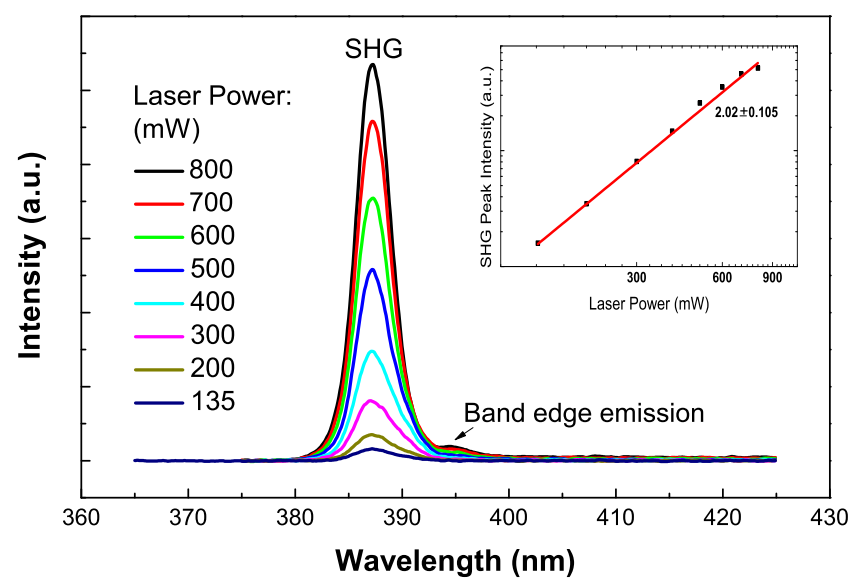

FIG. 3. (Color online) Emission spectra of a thin $\mathrm{ZnO}$ hollow rod under the excitation of $774 \mathrm{~nm}$ femtosecond laser measured at room temperature for different powers. The inset shows excitation-power dependence (solid squares) of the SHG signal intensity (logarithmic scale). The solid line in the inset figure is the fitting curve with a power index of $2.02 \pm 0.105$.

tion light, and $\theta$ : the angle between the excitation light beam and emission collection direction. Variable-excitation-power SHG measurements were carried out on the thin hollow rod at room temperature for different excitation wavelengths. Enhancement of the SHG emission was observed for a broad wavelength range of $700-860 \mathrm{~nm}$. Figure 3 shows the emission spectra of the sample under the excitation of femtosecond pulses with the wavelength of $774 \mathrm{~nm}$ for different powers. As shown in the inset of Fig. 3, the intensity of the SHG emission shows an almost ideal quadratic dependence on the excitation power. For example, the power index obtained from a least-squares fitting is $2.02 \pm 0.105$, which is very close to the ideal value of 2 . To conduct a further investigation on the SHG emission from the sample, we measured dependence of the SHG signal on the polarization of excitation beams with $732 \mathrm{~nm}$ and $740 \mathrm{~nm}$, respectively. The experimental data (solid squares) are shown in Fig. 4 for the excitation wavelength of $732 \mathrm{~nm}$. Dependence of the incoherent band-edge emission signal (open circles) on the polarization of the incident excitation beam was also measured and is illustrated in Fig. 4. Very similar results were measured for the excitation wavelength of $740 \mathrm{~nm}$ and are not shown here.



FIG. 4. (Color online) Measured dependence of integrated intensity of the SHG signal (solid squares) and band-edge emission (open circles) on the polarization of the excitation beam with wavelength of $732 \mathrm{~nm}$. The solid line shows the theoretical polarization dependence of the SHG signal. 


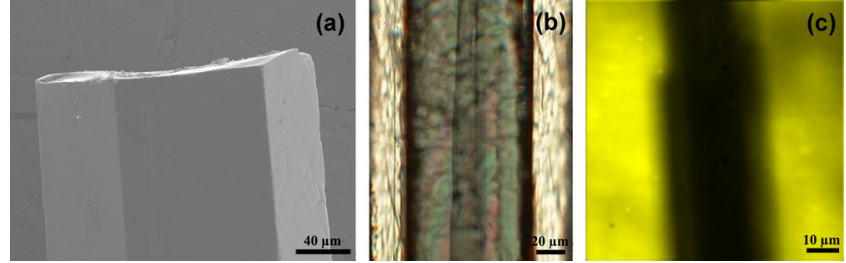

FIG. 5. (Color online) (a) SEM image of one end of a thin $\mathrm{ZnO}$ hollow rod with an excellent hexagonal shape; (b) optical microscopic image of the sample; (c) confocal micro-Raman image of the sample for a Raman shift range of $420-477.5 \mathrm{~cm}^{-1}$. Both the optical and Raman images consistently show the distinct hollow structures of the sample.

It is known that intensity of SHG signal from a single crystal is proportional to the square of second-order polarization induced by the fundamental beam, ${ }^{19,20}$

$$
I_{\mathrm{SHG}} \propto\left[P^{(2)}\left(\omega=2 \omega_{0}\right)\right]^{2},
$$

where $P^{(2)}\left(\omega=2 \omega_{0}\right)=\chi_{i j k}^{(2)} E_{j} E_{k}, \chi_{i j k}^{(2)}$ is the second-order susceptibility of the crystal. Since wurtzite $\mathrm{ZnO}$ crystal belongs to the $C_{6 v}$ (or $6 \mathrm{~mm}$ ) point group symmetry, and under Kleinman symmetry conditions, its two nonzero second-order susceptibility elements are: $\chi_{z z z}^{(2)}=-14.31 \mathrm{pm} / \mathrm{V}$, and $\chi_{z x x}^{(2)}$ $=1.36 \mathrm{pm} / \mathrm{V}^{21}$ As mentioned earlier, the incident angle of excitation beam is $\sim 26^{\circ}$ in the present study. So, the secondorder susceptibility as a function of the linear polarization angle $\varphi$ of the incident fundamental beam can be derived as,

$$
P^{(2)}\left(\omega=2 \omega_{0}\right) \propto\left(0.26 \sin ^{2} \varphi-14.31 \cos ^{2} \varphi\right) .
$$

Calculated dependence (solid line) of ideal SHG signal on polarization of incident fundamental beam using Eqs. (1) and (2) is shown in Fig. 4. It can be seen that a good agreement between experiment and theory is achieved. The polarization dependence of the band-edge emission of the $\mathrm{ZnO}$ tube studied in the present work is consistent with the previous report on $\mathrm{GaN}$ epilayers. ${ }^{19}$

In order to understand the enhancement mechanism of the SHG emission in the thin $\mathrm{ZnO}$ crystal rods, we did further structural characterizations of the samples. The SEM image in Fig. 5(a) shows that the thin rod possesses a characteristic hexagonal shape. By naked eyes, the reference thick rod sample has the similar hexagonal shape. Both the XRD data and SEM image indicate that the morphology and crystal structure of the thin $\mathrm{ZnO}$ rod have no difference from those of the thick rod sample. On the other hand, common optical microscopy and confocal micro-Raman microscopy observations reveal that the thin $\mathrm{ZnO}$ rod samples have distinct hollow geometric structures. The central hollow structures of the rods vary in a diameter range of $\sim 0.01-0.03 \mathrm{~mm}$ from sample to sample. All these $\mathrm{ZnO}$ narrow tubes studied in this work exhibit very efficient SHG emission. Figures 5(b) and 5(c) show the common optical microscopic image and micro-Raman image of such a thin rod sample, respectively. The window Raman frequency shift range of the Raman image is taken from 420 to $477.5 \mathrm{~cm}^{-1}$, which corresponds to the strongest Raman mode $\left(436 \mathrm{~cm}^{-1}\right.$, $E_{2}^{\text {high }}$ ) of wurtzite $\mathrm{ZnO}$ single crystal in the present study. The hollow geometric structures of the thin rods consistently shown by both the common optical microscopic and confo- cal micro-Raman images are believed to be responsible for the observed enhancement of SHG emissions from these samples.

It is known that nonlinear susceptibility of one sample determines how strong it makes response to incident light. ${ }^{22}$ For the wurtzite $\mathrm{ZnO}$ thin tubes studied in this work, their second order nonlinear susceptibilities shall include the surface part and bulk part which is due to the lack of central inversion symmetry in hexagonal crystalline structure. Compared with solid rods, hollow rods have an additional inner hollow surface which may contribute to the surface part of second order nonlinear susceptibilities. On the other hand, multiple total reflections of the transmitted part of incident primary beam most likely happen between the outer surface and inner hollow surface of the samples since the critical angle of total reflection for $800 \mathrm{~nm}$ light from $\mathrm{ZnO}$ into air is about $30^{\circ}$ (The refractive index of $\mathrm{ZnO}$ is taken as 1.96 at $800 \mathrm{~nm}$ ). The angle between the incident excitation light beam and collection direction of the SHG signal is $\sim 26^{\circ}$, as shown in the inset of Fig. 2. The optical signals are likely to be confined within the transverse section of the hollow rod. A simple quantitative estimation of the enhancement of the SHG in the thin hollow rod was done by considering the total reflection of the fundamental wave between the outer surface and inner surface inside the sample.

Again by using the well-established principles of nonlinear optics, contribution from the surface to SHG signal could be expressed as, ${ }^{19}$

$$
I_{S}(2 \omega) \propto\left|\chi_{S, \mathrm{eff}}^{(2)}\right|^{2} \cdot I^{2}(\omega),
$$

where $\chi_{S, \text { eff }}^{(2)}$ is the effective surface second-order susceptibility. Due to the total refection happening between the inner and outer surfaces of the sample, the surface contribution to the SHG signal should be amplified. The total reflection times was simply estimated to be at least three times within the focus region of the primary beam on the sample. Therefore, according to Eq. (3), the enhancement factor of the SHG intensity from the surface contribution due to the threetimes multiple total reflections shall be 9 . That is why so efficient SHG effect was observed in the $\mathrm{ZnO}$ hollow rod.

\section{SUMMARY}

To summarize, we observed an efficient SHG emission from thin $\mathrm{ZnO}$ hollow rods at room temperature. Structure characterizations and analysis reveal that multitotal reflections between the outer surface and inner hollow surface of the tubelike samples should be responsible for the observed efficient SHG emissions from the samples. The results presented in this work show that tubelike $\mathrm{ZnO}$ rods may have prospective applications in fabricating nonlinear optical devices.

\section{ACKNOWLEDGMENTS}

This work was supported by HK-RGC-GRF grants (Grant No. HKU705606P) and partially by PolyU500407P and HK-RGC-AoE Grant (Grant No. P-03/08). 
${ }^{1}$ Ü. Özgür, Ya. I. Alivov, C. Liu, A. Teke, M. A. Reshchikov, S. Doğan, V. Avrutin, S.-J. Cho, and H. Morkoç, J. Appl. Phys. 98, 041301 (2005).

${ }^{2}$ S. J. Chen, Y. C. Liu, C. L. Shao, R. Mu, Y. M. Lu, J. Y. Zhang, D. Z. Shen, and X. W. Fan, Adv. Mater. 17, 586 (2005).

${ }^{3}$ J. S. Jie, G. Z. Wang, Y. M. Chen, X. H. Han, Q. T. Wang, B. Xu, and J. G. Hou, Appl. Phys. Lett. 86, 031909 (2005).

${ }^{4}$ S. J. Xu, S. J. Xiong, and S. L. Shi, J. Chem. Phys. 123, 221105 (2005).

${ }^{5}$ A. M. Fischer, S. Srinivasan, R. Garcia, F. A. Ponce, S. E. Guano, B. C. Di Lello, F. J. Moura, and I. G. Solorzano, Appl. Phys. Lett. 91, 121905 (2007).

${ }^{6}$ H. Cao, J. Y. Wu, H. C. Ong, J. Y. Dai, and R. P. H. Chang, Appl. Phys. Lett. 73, 572 (1998).

${ }^{7}$ G. Wang, G. T. Kiehne, G. K. L. Wong, J. B. Ketterson, X. Liu, and R. P. H. Chang, Appl. Phys. Lett. 80, 401 (2002)

${ }^{8}$ X. Q. Zhang, Z. K. Tang, M. Kawasaki, A. Ohtomo, and H. Koinuma, Thin Solid Films 450, 320 (2004).

${ }^{9}$ S. K. Das, M. Bock, C. O'Neill, R. Grunwald, K. M. Lee, H. W. Lee, S. Lee, and F. Rotermund, Appl. Phys. Lett. 93, 181112 (2008).

${ }^{10}$ B. P. Mehl, R. L. House, A. Uppal, A. J. Reams, C. Zhang, J. R. Kirschbrown, and J. M. Papanikolas, J. Phys. Chem. A 114, 1241 (2010).

${ }^{11}$ M. C. Larciprete, D. Haertle, A. Belardini, M. Bertolotti, F. Sarto, and P. Gunter, Appl. Phys. B: Lasers Opt. 82, 431 (2006).
${ }^{12}$ B. Kulyk, Z. Essaidi, J. Luc, Z. Sofiani, G. Boudebs, B. Sahraoui, V. Kapustianyk, and B. Turko, J. Appl. Phys. 102, 113113 (2007).

${ }^{13}$ D. C. Dai, S. J. Xu, S. L. Shi, M. H. Xie, and C. M. Che, Opt. Lett. 30, 3377 (2005).

${ }^{14}$ D. C. Dai, S. J. Xu, S. L. Shi, M. H. Xie, and C. M. Che, IEEE Photonics Technol. Lett. 18, 1533 (2006).

${ }^{15}$ C. F. Zhang, Z. W. Dong, G. J. You, and S. X. Qian, Opt. Lett. 31, 3345 (2006).

${ }^{16}$ S. Zhang, J. Wang, S. Yao, R. I. Boughton, C. Zheng, and S. Xu, J. Chin. Ceram. Soc. 38, 338 (2010).

${ }^{17}$ N. Ashkenov, B. N. Mbenkum, C. Bundesmann, V. Riede, M. Lorenz, D. Spemann, E. M. Kaidashev, A. Kasic, M. Schubert, M. Grundmann, G. Wagner, H. Neumann, V. Darakchieva, H. Arwin, and B. Monemar, J. Appl. Phys. 93, 126 (2003).

${ }^{18}$ R. Cuscó, E. Alarcón-Lladó, J. Ibáñez, L. Artús, J. Jiménez, B. G. Wang, and M. J. Callahan, Phys. Rev. B 75, 165202 (2007).

${ }^{19}$ Y. R. Shen, The Principles of Nonlinear Optics (Academic, New York, 1993).

${ }^{20}$ H. Yang, S. J. Xu, and Q. Li, Appl. Phys. Lett. 88, 161113 (2006).

${ }^{21}$ G. Wang, G. K. L. Wong, and J. B. Ketterson, Appl. Opt. 40, 5436 (2001).

${ }^{22}$ Y. R. Shen, Appl. Phys. B: Lasers Opt. 68, 295 (1999). 